\title{
Crystal structure of aT cell receptor bound to an allogenic MHC molecule
}

Jean-Baptiste Reiser et al.

Nature Immunol. 1, 291-297 (2000).

In the October 2000 issue of Nature Immunology Figures 3 and 5 were printed incorrectly, although the online version and PDF are correct. Below are the correct figures.

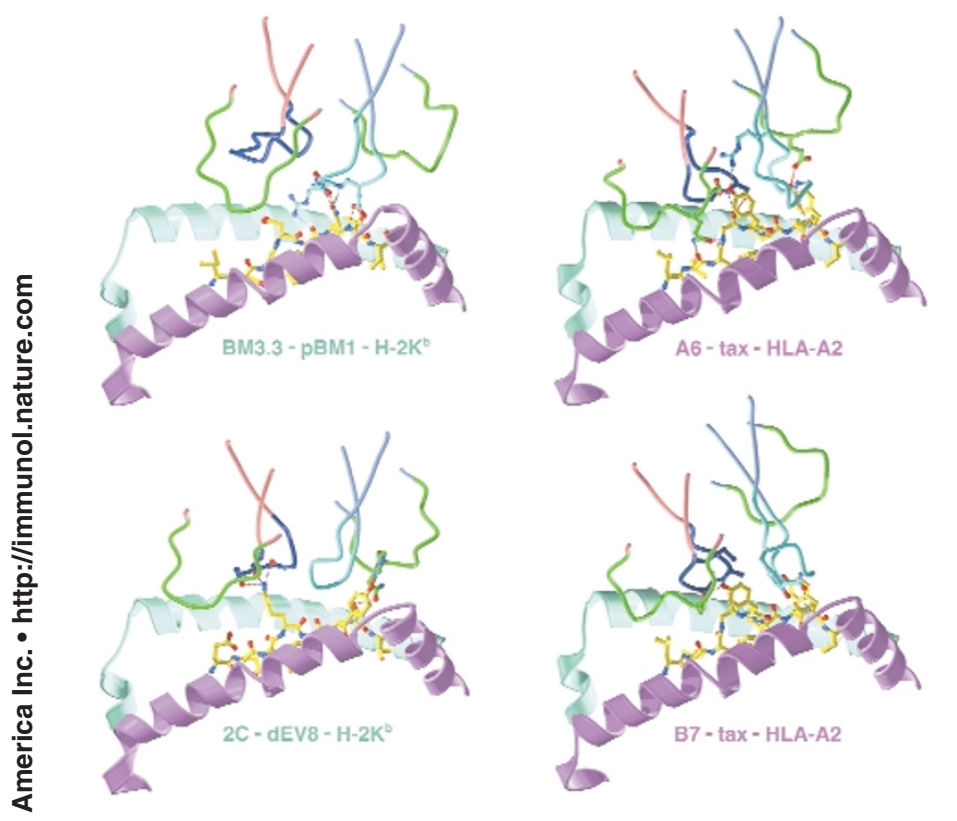

Figure 3. Peptide-TCR interactions occurring at the interface of fourTCRpMHC class I complexes. In this representation, the TCR-pMHC complex is viewed from the side so that the MHC $\alpha 2$-helix (light purple) is in the foreground and the MHC $\alpha 1$-helix (green) is behind the peptide (yellow). CDR2s have been removed for clarity. CDR1 and CDR3 are shown as a backbone worm diagram and color-coded as follows: CDR1 $\alpha$ and $\beta$ (green), CDR3 $\alpha$ (dark blue), CDR3 $\beta$ (cyan). The peptide residues involved in contact with the TCR are shown in ball-and-stick format. The corresponding hydrogen bonds are drawn as dotted lines. This representation highlights the different bends adopted by the tip of the CDR3 $\beta$ loop according to the length and conformation of the MHC-bound peptide.
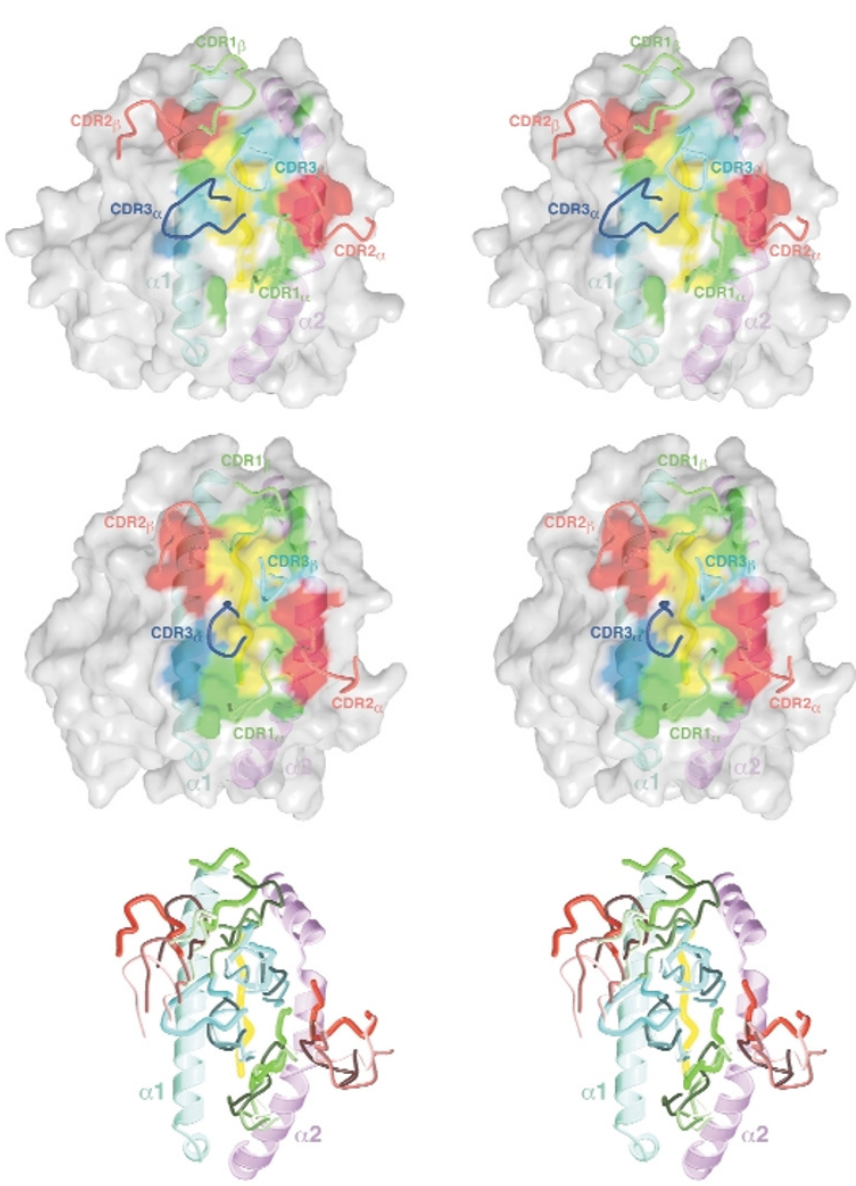

Figure 5. Docking ofTCRs to pMHC class I ligands. All three stereoviews are looking directly onto the surface of the peptide- $\mathrm{H}-2 \mathrm{~K}^{\mathrm{b}}$ complex; in each, the $\mathrm{C}$-terminus of the peptide is at the top. In (a) and (b), stereoviews represent the footprint of the molecular surface of the BM3.3 and $2 \mathrm{C}$ TCR binding sites on the peptide $\mathrm{H}-2 \mathrm{~K}^{\mathrm{b}}$ solvent-accessible surface, respectively. The $\mathrm{H}-2 \mathrm{~K}^{\mathrm{b}}$ surface is color-coded according to the CDRs that contact it: CDR1-buried (green), CDR2-buried (red), CDR3 $\alpha$-buried (blue) and CDR3 $\beta$-buried (cyan).The CDRs are represented as thin worms using the same color code.The peptide backbone (thick worm) and its solvent-accessible surface are depicted in yellow. The $\mathrm{H}-2 \mathrm{~K}^{\mathrm{b}} \alpha$-helices are visible through the semitransparent surface and colored in light emerald green $(\alpha 1)$ or light purple $(\alpha 2)$. In the stereoview shown in (c), the $\alpha 1 / \alpha 2$-helices of the MHC component found in various TCR-pMHC class I complexes were superimposed to highlight the differences occurring between the respective CDR footprints on pMHC class I ligands.The MHC $\alpha$-helices and the CDRs have been drawn using the same color code as in $\mathbf{a}$ and $\mathbf{b}$. The CDRs of the different TCRs are coded as follows: BM 3.3 (thicker worm and fully saturated colors), 2C (dark colors), A6 (medium intensity colors) and B7 (pale colors). 